\title{
Complémentarité des mesures nucléaires et de spectrométrie de masse, dans le cadre de l'analyse radiotoxicologique. Application aux urines et fèces
}

\author{
N. BAGLAN*, A. AMARAL*. C. COSSONNET*, \\ D. FRANCK*, J. RITT*
}

(Manuscrit reçu le 17 mars 1997, révisé le 15 juillet 1997, accepté le 8 septembre 1997)

RÉSUMÉ La méthode utilisée en routine pour déterminer une éventuelle contamination par des actinides, émetteurs $\alpha$, est la mesure de la fraction éliminée dans les excreta (urine et/ou feces). La procédure actuelle se compose de deux étapes, tout d'abord une séparation radiochimique sélective de chaque élément présent puis, après préparation d'une source électrodéposée, la mesure de son activité par spectrométrie $\alpha$. Dans un souci de simplification de cette procédure, on évalue ici les performances de l'analyse des actinides par spectrométrie de masse, pour deux techniques particulières l'ICP-MS et le SIMS. L'utilisation des trois outils analytiques (spectrométrie $\alpha$, ICP-MS, SIMS) est étudiée sur la base de résultats récents provenant de la littérature et de ceux obtenus dans le laboratoire. Sur la base de ces résultats, le présent article analyse les avantages propres à chaque technique.

ABSTRACT Complementarity of nuclear spectrometry and multielements techniques for radiotoxicological analysis. Application to urine and faeces.

The method routinely used to determine a possible contamination by actinides, alpha emitters, is the measurement of the fraction transferred to the excreta (urine and/or faeces). The first step of the procedure commonly used consists in a radiochemical specific separation of each element. In the second step an electroplated source is prepared to allow the measurement of its activity by alpha spectrometry. In order to simplify this procedure, the performance of the analysis of actinides by mass spectrometry is estimated based on two analytical tools, the ICP-MS and the SIMS. The use of the three techniques ( $\alpha$ spectrometry, ICP-MS, SIMS) is investigated based on recent results published in the literature or research work carried out in the laboratory. The results reported in this study allow to compareassess the advantages of each technique. 


\section{Introduction}

Dans le cadre de la surveillance des travailleurs du nucléaire, l'évaluation quantitative du degré de contamination suite à l'ingestion et/ou l'inhalation accidentelle d'émetteurs alpha reste un problème difficile à résoudre. En effet, contrairement aux photons, le parcours des particules $\alpha$ émises dans l'organisme est insuffisant pour permettre leur mesure directe. On est ainsi conduit à effectuer la surveillance individuelle en mesurant l'activité éliminée par l'organisme. Les analyses des excreta (urine, fèces) sont utilisées comme données pour déduire l'activité initialement incorporée et celle présente dans le corps à partir de modèles biocinétiques. Cependant, même si cette approche fondée le plus généralement sur la mesure d'échantillons en couches minces par spectrométrie alpha, est bien établie, elle rencontre des difficultés principalement liées à la présence simultanée de plusieurs éléments ( $\mathrm{Th}, \mathrm{U}, \mathrm{Pu}$, Am). Une séparation radiochimique (Harduin et al., 1996) lourde est indispensable pour parvenir à mesurer sans interférence l'activité de chacun d'eux. De plus, une fois les sources électrodéposées réalisées, les temps de comptage nécessaires pour atteindre les limites préconisées $(1 \mathrm{mBq})$ sont longs.

Les Laboratoires d'Analyses et de Biologie Médicale (LABM) ont en charge la surveillance en routine des travailleurs ainsi que l'évaluation des contaminations aiguës en cas d'accident. Dans ce cadre, des procédés d'analyse de mise en œuvre simple et rapide ainsi que l'utilisation de faibles quantités d'échantillons présentent un intérêt certain. Pour atteindre cet objectif, la recherche de la simplification des procédés d'analyse radiotoxicologique doit porter à la fois sur les méthodes de purification ainsi que sur les techniques de détection des actinides. Dans ce cadre deux axes de recherche sont poursuivis :

- le développement de procédés chimiques d'extraction plus sélectifs;

- le développement de l'analyse d'échantillons biologiques par spectrométrie de masse.

Le premier axe étant décrit par ailleurs (Dinse et al., 1997) cet article traite plus spécifiquement du développement de l'analyse par spectrométrie de masse. Deux techniques sont étudiées, selon les cas alternatives ou complémentaires à la spectrométrie alpha :

- l'ICP-MS «Inductively Coupled Plasma-Mass Spectrometry » plus précisément choisie étant donné son intérêt possible pour l'analyse radiotoxicologique;

- la technique SIMS « Secondary Ion Mass Spectrometry », dédiée à l'analyse de solide est caractérisée par une très bonne résolution, ce qui la rend plus performante pour la mesure d'isotopie. Dans le cadre de cette étude, son application à des solutions a nécessité la préparation d'une source homogène, constituée d'un film polymère déposé sur une plaque d'or, sur laquelle, la fixation 
des actinides se fait par échange d'ions avec le polymère (Stéphan et al., 1997). La technique SIMS reste dans un premier temps envisagée comme technique d'expertise et de recherche.

Le présent article passe en revue les principales caractéristiques des trois techniques de mesure et compare leur niveau d'application dans la perspective de l'analyse radiotoxicologique pour la surveillance individuelle (limite de détection, mesure de rapport ou de nuance isotopique, rapidité, quantité d'échantillons, etc.).

\section{Description des techniques analytiques}

\subsection{Spectrométrie alpha}

\subsubsection{Principe}

L'énergie des particules $\alpha$ émises est mesurée à l'aide d'un détecteur à semi-conducteur. Ce système permet de convertir l'énergie cédée par les particules chargées, en impulsions dont l'amplitude est proportionnelle à l'énergie cédée au détecteur. En classant ces impulsions en fonction de leur amplitude pendant un temps suffisamment long, on obtient le spectre d'énergie alpha de la source électrodéposée. Pour minimiser les pertes d'énergie des particules alpha entre la source et le détecteur, il est nécessaire de faire le vide dans la chambre de mesure.

\section{TABLEAU 1}

Limites de détection pour certains actinides en spectrométrie alpha.

Detection limit for some actinides measurement by alpha spectrometry.

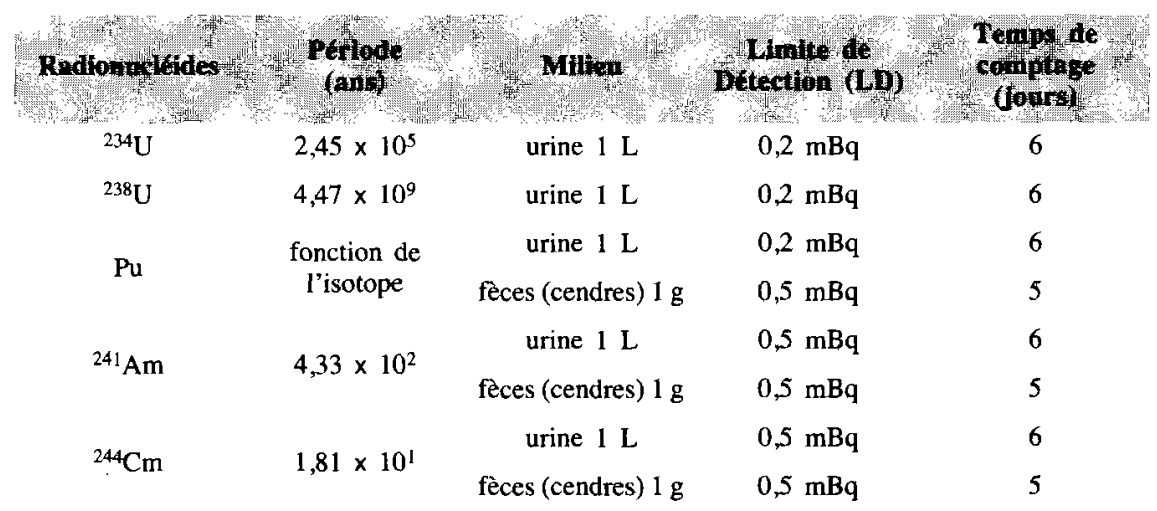




\subsubsection{Performances et gamme d'application}

Le tableau I résume les performances obtenues par spectrométrie alpha dans le laboratoire pour mesurer les actinides dans les urines et fèces. Après séparation chimique, la plupart des interférences spectrales sont résolues. Les limites de détection(1) qu'il est possible d'atteindre, évaluées de la façon suivante :

$$
\mathrm{LD}=\frac{5,66(\sqrt{B})}{e R t}
$$

sont suffisamment basses pourvu que les temps de comptage soient assez longs.

$B$ : bruit de fond pendant le temps $t$ pris sur le même nombre de canaux que celui utilisé pour l'évaluation de l'activité de l'élément considéré (imp)

$e$ : efficacité

$R$ : rendement chimique

$t$ : temps de comptage (s).

La spectrométrie alpha est utilisée très largement dans le domaine de la mesure d'actinides à bas niveau et ceci quelle que soit leur origine (cycle du combustible, échantillons de l'environnement). On notera surtout que, malgré tous les inconvénients liés à la préparation des sources, à la durée des comptages, elle reste la méthode de référence pour l'analyse radiotoxicologique. Au niveau des LABM, les contraintes liées à la surveillance des travailleurs ne permettent pas des temps de comptage aussi longs. Typiquement, les sources alpha sont comptées trois jours, avec une limite de détection de l'ordre du $\mathrm{mBq}$, tout à fait en accord avec celle du laboratoire pour un temps de comptage équivalent.

\subsection{ICP-MS (Inductively Coupled Plasma Mass Spectrometry)}

\subsubsection{Principe}

L'analyse des échantillons réels, de part la variété des matrices ${ }^{(1)}$ et des éléments constitutifs, nécessite la mise au point d'instruments permettant des déterminations multiélémentaires. Ces appareils sont constitués d'une part d'un système d'excitation et, d'autre part, d'un système d'analyse et de détection. Les pionniers ont utilisé l'arc électrique associé à un spectrographe puis à un spectromètre. À la fin des années 70 vinrent les systèmes remplaçant l'arc par un plasma inductif (ICP-AES) ou conductif (DCP-AES)

(1) La définition de tous les termes repérés par ce symbole sont définis dans un glossaire en fin d'article. 
associé à un spectromètre d'émission atomique. Depuis une dizaine d'années, afin d'améliorer la sensibilité(1), l'ensemble analytique est remplacé par un spectromètre de masse (ICP-MS). Ce dernier couplage trouve tout son intérêt, d'une part, dans les bonnes performances du plasma inductif :

- une température élevée au sein de celui-ci (jusqu'à $8000 \mathrm{~K}$ ),

- l'utilisation de l'argon, d'énergie d'ionisation élevée $(15,6 \mathrm{eV})$, offrant la possibilité d'ioniser la plupart des éléments du tableau périodique et assurant un milieu inerte,

- peu d'interférences chimiques ou physico-chimiques, et, d'autre part, dans celles du spectromètre de masse :

- l'utilisation d'un filtre quadripolaire de résolution limitée (environ $1 \mathrm{u}$ ) mais suffisante surtout pour les masses supérieures à 80 u où les interférences polyatomiques sont beaucoup moins importantes,

- un système fiable car ne comportant pas de pièce en mouvement.

\section{TABLEAU II}

Limites de détection obtenues en ICP-MS dans la présente étude pour la mesure de ${ }^{238} \mathrm{U}$.

Detection limit obtained in the present study by ICP-MS for ${ }^{238} \mathrm{U}$.

$\left({ }^{238} \mathrm{U} 1 \mathrm{ppq}=12,44 \times 10^{-6} \mathrm{mBq}\right)$.

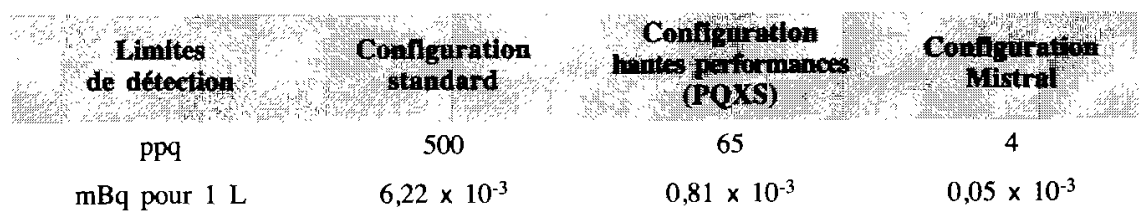

\subsubsection{Performances et gamme d'application}

Le tableau II résume les performances de l'ICP-MS obtenues au laboratoire pour l'uranium dans des solutions étalons. Les différentes configurations citées sont :

- la configuration standard (PQXR),

- la configuration hautes performances, où une amélioration du vide, donc de la transmission des ions permet d'améliorer la sensibilité,

- la configuration Mistral, qui combine l'amélioration du vide avec un module d'introduction de l'échantillon plus performant. 
TABLEAU III

Définition des unités usuelles en ICP-MS.

Definition of the unity currently used in ICP-MS.

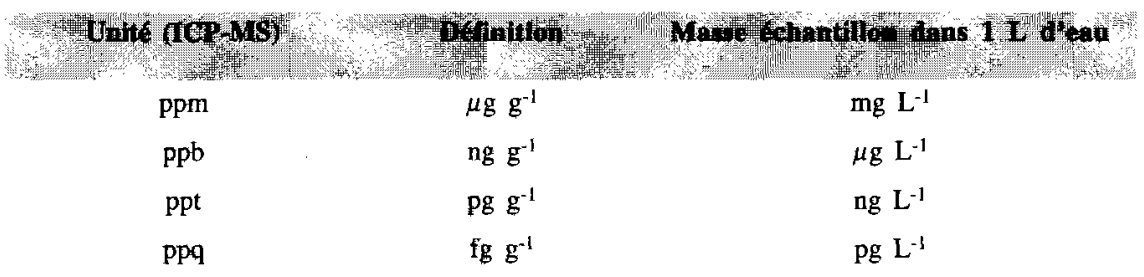

Tout d'abord les résultats sont exprimés dans les unités usuelles en ICPMS, la ppm et ses sous multiples, définies dans le tableau III, puis en introduisant l'activité spécifique de l'uranium 238 en $\mathrm{Bq} \mathrm{g}^{-1}$. On notera cependant que les LD effectives dépendront in fine de manière critique de chaque type d'échantillon et des matrices réelles à analyser.

L'utilisation de l'ICP-MS, qui a connu un essor considérable durant les dix dernières années est maintenant largement répandue dans certains domaines comme la mesure des actinides à vie longue où les deux pôles essentiels sont les échantillons de l'environnement et les impuretés dans la matrice d'uranium. Par contre, son utilisation dans le domaine de l'analyse radiotoxicologique a été envisagée plus récemment (Wyse et al., 1994; Werner et al., 1996; Nguyen et al., 1996).

\subsection{SIMS (Secondary Ion Mass Spectrometry)}

\subsubsection{Principe}

Le phénomène d'émission ionique secondaire a été mis à profit par Castaing et Slodzian (1960) pour la construction du microanalyseur ionique. Dans celuici, les ions secondaires sont arrachés de la surface de l'échantillon sous l'action du bombardement des ions primaires et sont émis avec une énergie relativement faible : on les accélère au moyen d'une lentille électrostatique qui les focalisent en formant une image globale et agrandie de la surface de l'échantillon. Le spectromètre de masse décompose cette image globale en autant d'images élémentaires qu'elle comporte de particules ionisées de masses différentes. Un convertisseur d'image restitue chaque image élémentaire sur un écran ou une caméra haute résolution. À côté de ses capacités d'imageur, le SIMS est un analyseur de haute performance. Au lieu de prendre un cliché à une masse donnée, on peut faire varier continûment le champ magnétique et enregistrer les courants ioniques correspondant aux masses successives. L'intérêt de cette méthode de microanalyse est une exploration 
systématique de l'ensemble de la classification périodique. La technique est destructive, propriété qui peut cependant être mise à profit pour l'étude des variations de concentration en fonction de la profondeur.

\subsubsection{Performances et gamme d'application}

Les applications les plus courantes sont rapportées dans les domaines suivants (Morrison et al., 1975) : géologie, micro-électronique, métallurgie (Degrève et al., 1988), physico-chimie, biologie (Berry et al., 1986). Pour les analyses radiotoxicologiques, on ne dispose d'aucune référence relative à l'application du SIMS pour la mesure de l'uranium et du plutonium. Les premières valeurs présentées dans le tableau IV montrent les performances obtenues dans la présente étude pour les actinides (U et $\mathrm{Pu}$ ) dans l'urine (Amaral et al., 1996).

\section{TABLEAU IV}

Limite de détection obtenue pour la mesure de ${ }^{238} \mathrm{U}$ et ${ }^{239} \mathrm{Pu}$ par la technique SIMS. Minimum detectable activity obtained for the measurement of ${ }^{238} \mathrm{U}$ et ${ }^{239} \mathrm{Pu}$ using the SIMS.

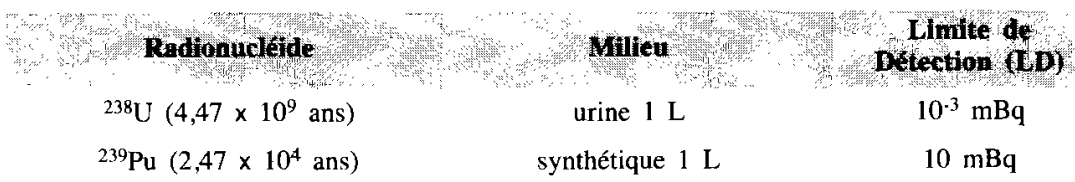

\section{TABLEAU $V$}

Comparaison des trois techniques analytiques.

Comparison of the three analytical techniques.

\begin{tabular}{|c|c|c|c|c|c|c|}
\hline Technique & Eléments & Speciation & Résolution" & Isotopie & $\begin{array}{l}\text { Effet de } \\
\text { matriee }\end{array}$ & $\begin{array}{c}\text { LD pour }{ }^{238} \mathrm{U} \\
(\text { m Bq) }\end{array}$ \\
\hline Spectrométrie $\alpha$ & émetteurs $\alpha$ & non & faible & oui & fort & $2,0 \times 10^{-1}$ \\
\hline SIMS & tous & non & bonne & oui & fort & $1,0 \times 10^{-3}(\$)$ \\
\hline ICP-MS & tous & non & moyenne & oui & moyen & $8,1 \times 10^{-4}\left({ }^{*}\right)$ \\
\hline
\end{tabular}

(\#) limite de détection calculée pour ${ }^{238} \mathrm{U}$ dans l'urine.

(\$) limite de détection calculée pour ${ }^{238} \mathrm{U}$ dans l'urine.

${ }^{*}$ ) limite de détection calculée pour ${ }^{238} \mathrm{U}$ dans une solution étalon.

\section{Comparaison des techniques analytiques}

Les performances globales des trois techniques citées sont récapitulées dans le tableau V. Les limites de détection sont données en atomes par litre pour les deux techniques élémentaires, ce qui permet une conversion rapide en activité ou en concentration pour plusieurs isotopes d'un même élément. De plus ce 
mode d'expression du résultat met bien en évidence l'influence de la période sur la sensibilité de ces techniques. Pour l'uranium 238 les valeurs de LD accessibles pour l'ICP-MS et le SIMS sont comprises entre respectivement $5 \times 10^{-5}$ et $10^{-3} \mathrm{mBq}$ (comparé à $2 \times 10^{-1} \mathrm{mBq}$ en spectrométrie $\alpha$ ).

\section{TABLEAU VI}

Comparaison des limites de détection observées en spectrométrie alpha et pour l'ensemble Mistral/ICP-MS (SMSRB).

Comparison between the detection limits obtained by a spectrometry and the sample introduction device Mistral coupled with ICP-MS (SMSRB)

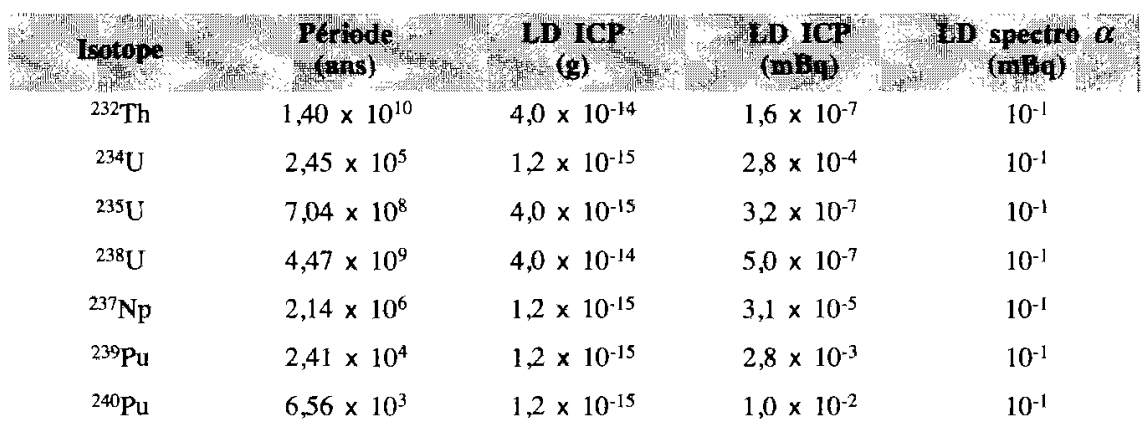

Les valeurs des LD seront certainement plus faibles pour tous les isotopes artificiels de l'élément. En effet dans toutes les solutions utilisées, même les blancs ou les réactifs, une pollution d'uranium naturel (ou appauvri) existe diminuant ainsi la sensibilité de l'ICP-MS pour les isotopes naturels. Ceci est confirmé par les résultats publiés par le SMSRB ${ }^{(2)}$ reportés dans le tableau VI (Chiappini et al., 1996).

On s'aperçoit, à la lecture de ce tableau, que les techniques élémentaires peuvent être beaucoup plus sensibles à partir de périodes de l'ordre de $10^{4}$ ans, voire même compétitives pour des périodes inférieures. Ces résultats, obtenus avec des solutions étalons, mettent en évidence le potentiel de l'outil analytique. Cependant, ces résultats devront être confirmés pour des analyses d'échantillons de type urine puis fèces, où les interférences liées à la matrice ${ }^{(1)}$ pourront entraîner une détérioration de ces performances.

(2) Service mixte de Surveillance Radiologique et Biologique de l'homme et de l'environnement Ministère de la défense/DIRCEN - CEA/DAM, Montlhéry. 


\section{Conclusion}

À ce jour, la spectrométrie alpha est la technique de référence pour la mesure d'émetteurs alpha dans les urines et fèces, de par sa simplicité d'emploi et son faible coût. En parallèle et avec les mêmes objectifs, l'utilisation de l'ICP-MS pour l'analyse de radionucléides à vie longue dans les excreta se développe dans des laboratoires pilotes comme le laboratoire de chimie analytique de Valduc (CEA-DAM). Une fois l'adéquation obtenue entre les solutions d'élution provenant du protocole de purification et les contraintes relatives à la charge saline maximale conseillées en ICP-MS (Ross et al., 1993), la mesure de l'uranium 238 ne pose pas de problèmes importants. Pour ce qui est du SIMS, la technique n'a, à ce jour, pas encore atteint une maturité suffisante pour une utilisation en routine.

\section{TABLEAU VII}

Synthèse des avantages et inconvénients des différentes techniques. Strengths and weaknesses related to each technique.

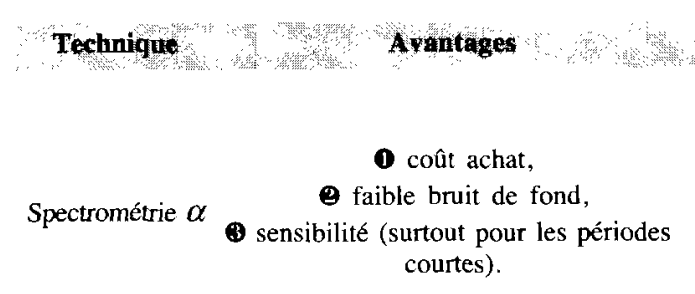

- rapidité,

0 rapidité,
9 large gamme d'utilisation potentielle
dynamique et massique,
3 possibilité de déterminer des rapports
isotopiques et de discriminer des masses
proches,
SIMS $\quad$ possibilité de réaliser des profils en
profondeur,
( choix de la source d'ions primaires
possible (taux d'ions secondaires est
fonction des natures respectives des ions
primaires et secondaires),
6 coût d'une analyse.

\section{Inconvelients}

- temps de comptage,

2 coût d'une analyse,

3 chimie séparative indispensable pour mesurer plusieurs actinides,

(- obligation de préparer une source sur support solide avant toute mesure,

Dcontributions du plutonium 239 et 240 indiscernables.

\section{(1) coût achat, \\ 3 méthode destructive,}

B obligation de préparer une source sur support solide avant toute mesure,

๑ interférences polyatomiques, liées à la matrice,

3 influence de ce support (type, maîtrise de l'épaisseur et de l'homogénéité du dépôt),

complexité de l'équipement.et de l'informatique 


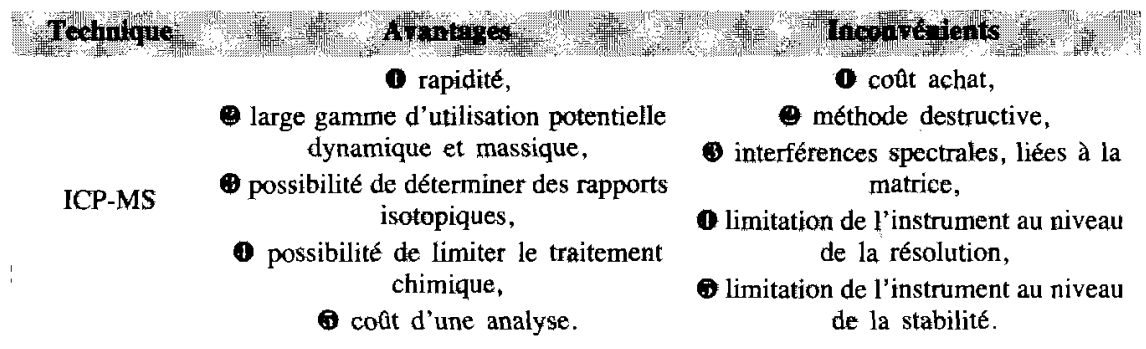

Un point important se doit d'être souligné, les techniques basées sur la spectrométrie de masse mesurent un nombre d'atomes. Si celui-ci reste constant, sur la gamme de masse étudiée, l'activité associée ira croissante avec la diminution de la période du radionuclèide considéré. Cependant ces techniques possèdent des avantages déterminants par rapport à la spectrométrie $\alpha$ (Tab. VII) :

1) la possibilité de détermination d'un rapport isotopique dont ${ }^{239} \mathrm{Pu} / 240 \mathrm{Pu}$,

2) la sensibilité pour les actinides de longue période (Tab. VI).

Toutefois, aucune des trois techniques ne procure de solution universelle. À ce jour, le SIMS, dans le cadre de l'analyse radiotoxicologique, reste une technique de recherche. Les deux autres techniques (ICP-MS et spectrométrie $\alpha$ ) sont utilisables en routine. Leur application présente différents avantages qu'il convient d'évaluer à la lumière des exigences posées par la surveillance individuelle : type de contamination, délai entre la contamination et la mesure, délai de réponse, niveau d'activité à atteindre, période du contaminant.

Remerciements. Nous remercions tout particulièrement MM. Chiappini et Taillade pour leurs conseils lors de la mise en ceuvre du laboratoire ICP-MS.

\begin{abstract}
ANNEXE
Limite de détection (LD): quantité minimale détectable d'un élément dans un échantillon qui sera détectée avec une probabilité $\beta$ de non détection (erreur de type II) en acceptant une probabilité $\alpha$ d'affirmer de manière erronée la présence d'élément à analyser dans le blanc correspondant (erreur de type I)

Matrice : environnement de l'élément à analyser.

Effet de matrice : influence des éléments constituant la matrice sur les performances de l'outil analytique.

Résolution : plus petit intervalle entre deux pics adjacents tel qu'ils puissent être discriminés par l'outil analytique.

Sensibilité : quotient de l'accroissement de la réponse d'un instrument de mesure par l'accroissement correspondant du signal d'entrée.

Interférence isobarique : contribution de deux éléments de même rapport $\mathrm{m} / \mathrm{z}$ non séparée par le spectromètre de masse.
\end{abstract}




\section{RÉFÉRENCES}

Amaral A., Galle P., Cossonnet C., Franck D., Pihet P., Carrier M., Stephan O. (1996) Perspective of uranium and plutonium analysis in urine sample by secondary ion mass spectrometry, soumis à J. of Radioanalytical Chemistry.

Berry J. P., Escaig F., Lange F., Galle P. (1986) Ion microscopy of the thyroid gland : a method for imaging stable and radioactive iodine, Laboratory investigation, 55-1, 109-119.

Castaing R.,Slodzian G. (1960) Microanalyse par émission ionique secondaire, J. Microscopie, I, 395410 .

Chiappini R., Taillade J. M., Brebion S. (1996) Development of a high sensitivity inductively coupled plasma mass spectrometer for actinide measurement in the femtogram range, J.A.A.S., 11,497503.

Degrève F., Thorne N. A., Lang J. M. (1988) Metallurgical applications of secondary ion mass spectrometry (SIMS), J. Mater. Sci., 23, 4181-4208.

Dinse C., Cossonnet C., Baglan N., Asfari Z., Vicens J., Extraction sélective des actinides, application à l'analyse radiotoxicologique, Radioprotection 32-5.

Harduin J. C., Peleau B., Levavasseur D. (1996) Analytical determination of actinides in biological samples, Radioprotection, 31-2, 229-245.

Morrison G. H., Slodzian G. (1975) Ion microscopy, Anal Chem., 47-11, 932A-943A.

Nguyen S. N., Miller P. E., Wild J. F., Hickman D. P. (1996) Simultaneous determination of ${ }^{237} \mathrm{~Np}$, ${ }^{232} \mathrm{Th}$ and $\mathrm{U}$ isotopes in urine samples using extraction chromatography, ICP-MS and gamma spectroscopy, Radioact. Radiochem., 7-3, 16-21.

Ross R. R., Noyce J. R., Lardt M. M. (1993) Inductively Coupled Plasma-Mass Spectrometry : An emerging method for analysis of long lived radionuclides, Radioact. Radiochem., 4-1, 24-36.

Stéphan O., Carrier M. (1997) Preparation of thin $\alpha$-particle sources using polypyrrole films functionalized by alkylammonium groups, Radiochim. Acta, 76, 29-36.

Werner E., Roth P., Wendler I., Schramel P. (1996) Assessment of thorium excretion in urine by means of ICP-MS. In : Proceedings IRPA9, 2, 489-491.

Wemer E., Roth P., Wendler I., Schramel P, Hellmann H., Kratzel U. (1996) Feasibility of ICP-MS for the assessment of uranium excretion in urine, soumis à $J$. Radioanal. Chem..

Wyse E. J., Fisher D. (1994) Radionuclide bioassay by inductively coupled plasma mass spectrometry (ICP-MS), Radiat. Prot. Dosim., 55-3, 199-206. 